\title{
A Case of Spontaneous Regression of Recurrent Desmoid Tumor Originating From the Internal Obturator Muscle After Delivery
}

\author{
Takuya Sueishi ${ }^{a}$, Takeshi Arizono ${ }^{a}$, b, Kenjiro Nishida ${ }^{a}$, Takahiro Hamada ${ }^{a}$, \\ Akihiko Inokuchi ${ }^{\mathrm{a}}$
}

\begin{abstract}
Desmoid-type fibromatoses are pathologically benign but locally aggressive tumors. We report the case of a desmoid tumor that disappeared spontaneously after recurrence. A 21-year-old woman was referred to our hospital because of left lower limb weakness during menstruation. The following day this weakness had disappeared but menstrual colic remained; consequently, the patient underwent an internal examination that revealed an intrapelvic tumor. Magnetic resonance imaging demonstrated an enhanced mass (diameter, $8 \mathrm{~cm}$ ) arising from the internal obturator muscle and attached to the urinary bladder. The tumor was diagnosed as a desmoid-type fibromatosis after histologic evaluation of a transvaginal biopsy; marginal resection was carried out at $<1$ month after the first hospital admission. The patient experienced recurrence at 2 years after surgery, which was confirmed as two enhanced masses (diameter, $1 \mathrm{~cm}$ ) using magnetic resonance imaging. Eleven months later, the diameters of these masses had increased to $1.8 \mathrm{~cm}$; however, there was no further increase in size beyond this point. The patient delivered successfully at 5 and 7 years after surgery; at 8 years, the recurrent tumors had disappeared completely as confirmed by magnetic resonance imaging. This case involving recurrence is rare for two reasons. The first was that no change in the size of the tumors occurred during pregnancy and after delivery, and the second was that the patient experienced complete remission of the recurrent tumors after only simple observation. Thus, it is important to recognize that even a recurrent desmoid tumor can sometimes exhibit spontaneous regression.
\end{abstract}

Keywords: Desmoid; Simple observation; Spontaneous regression

\section{Introduction}

The fibromatoses are a group of fibroblastic proliferations dis-

\section{Manuscript accepted for publication March 03, 2016}

aDepartment of Orthopedic Surgery, Kyushu-Central Hospital, 23-1 Shiobaru 3-chome, Minami-ku, Fukuoka 815-8588, Japan

${ }^{b}$ Corresponding Author: Takeshi Arizono, Department of Orthopedic Surgery, Kyushu-Central Hospital, 23-1 Shiobaru 3-chome, Minami-ku, Fukuoka 8158588, Japan. Email: arizono@kyushu-ctr-hsp.com

doi: http://dx.doi.org/10.14740/wjon963w tinguished by their tendency for local aggressiveness. They are divided into two major clinicopathologic groups, namely superficial and deep. The deep fibromatoses include the so-called desmoid tumors [1]. These are rare histologically benign tumors. Despite their benign appearance, they often exhibit repeat recurrences clinically. We report the case of a woman found to have a recurrent desmoid tumor located in the internal obturator muscle that disappeared spontaneously.

\section{Case Report}

The study was approved by the ethics committee of our hospital. The patient gave her informed consent prior to her inclusion in the study. A 21-year-old woman with no history of illness was referred to our hospital because of left lower limb weakness during menstruation. The following day the weakness had disappeared but the menstrual colic remained; consequently, the patient was referred to a gynecologist who detected an intrapelvic tumor during internal examination. The lesion was a palpable hard mass the size of a hen's egg located in the right vaginal wall. The patient's laboratory evaluation included tumor markers, which showed unremarkable scores. On magnetic resonance imaging, there was an enhanced mass $(8 \mathrm{~cm}$ in diameter) attached to the urinary bladder and arising from the internal obturator muscle (Fig. 1). The tumor showed a moderately low signal intensity comparable to that of the skeletal muscles on T1-weighted images, a moderately high signal intensity on T2-weighted images, and high enhancement with a partially mottled pattern after intravenous administration of gadolinium contrast agent. Histological diagnosis of the tumors by means of transvaginal biopsy was desmoid-type fibromatosis. Marginal resection was undertaken at $<1$ month after the first visit to our hospital. The tumor seemed to arise from the right internal obturator muscle behind the bladder; there was remarkable synechia to the rectum and retroperitoneum, but no invasion was evident on evaluation of surgical specimens. The specimens removed were $7-8 \mathrm{~cm}$ in diameter, reddish brown, and had hard elastic characteristics. Transverse sections had a yellow-white appearance. Pathological analysis revealed welldifferentiated fibroblasts, which had no dysplasia or abnormal fission proliferation, and had infiltrated the skeletal muscles; thus, it was diagnosed as a desmoid tumor (Fig. 2).

At 27 months after surgery, the patient developed recur- 

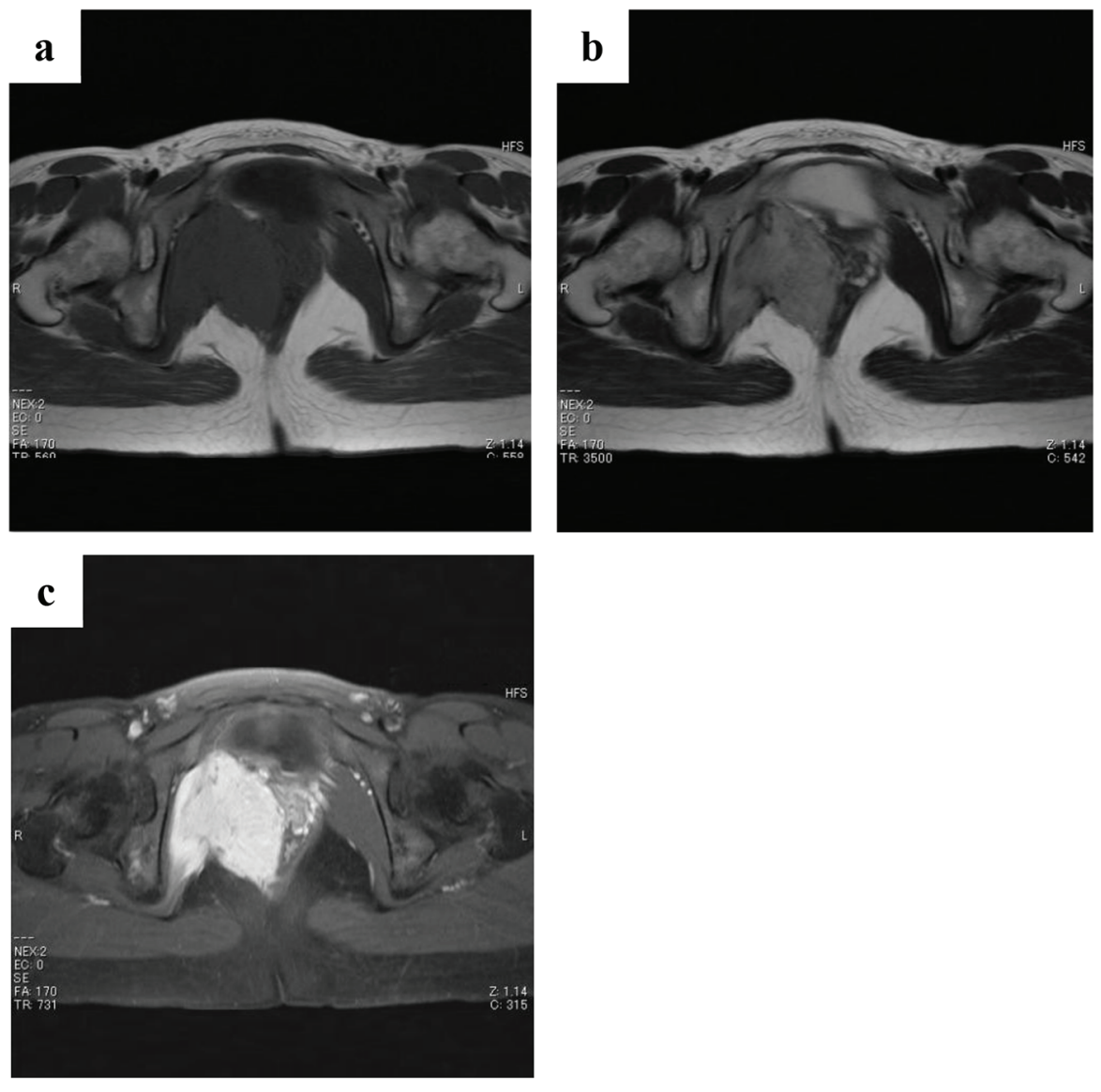

Figure 1. Magnetic resonance images obtained at first examination. There is an enhanced mass $(8 \mathrm{~cm}$ in diameter) attached to the urinary bladder arising from the internal obturator muscle. The tumor shows a moderately low signal intensity comparable to skeletal muscles on T1-weighted images (a), a moderately high signal intensity on T2-weighted images (b), and high enhancement with a partially mottled pattern after intravenous administration of gadolinium contrast agent (c).

rences that were confirmed by magnetic resonance imaging as two enhanced masses ( $1 \mathrm{~cm}$ in diameter) located a little behind the preoperative point, namely the right internal obturator muscle (Fig. 3). Eleven months later, these masses had increased in size to $1.8 \mathrm{~cm}$ in diameter (Fig. 4), but no further changes in size occurred after this time (Fig. 5). The patient delivered successfully at 5 and 7 years after surgery. At 8 years after surgery, the recurrent tumors had completely disappeared as confirmed by magnetic resonance imaging (Fig. 6).

\section{Discussion}

Desmoid tumors are neoplasms that are characterized by the


Figure 2. Pathological sections of a tissue specimen stained with hematoxylin and eosin. Well-differentiated fibroblasts that do not exhibit dysplasia or abnormal fission proliferation have infiltrated the skeletal muscles. 

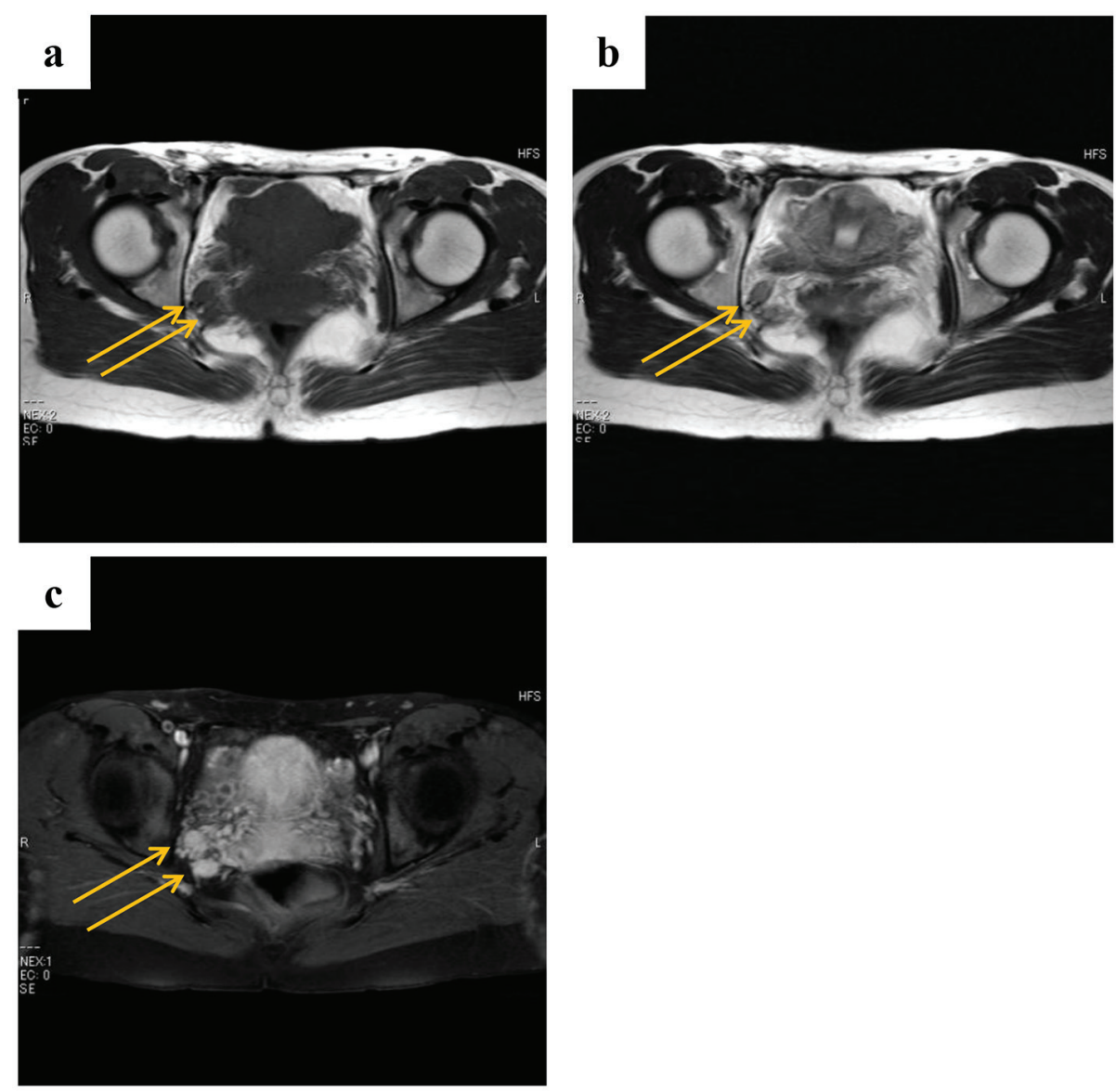

Figure 3. Magnetic resonance images obtained at 2 years and 3 months after surgery (a: T1-weighted images; b: T2-weighted images; c: gadolinium contrast). The patient had tumor recurrence that was evident as two enhanced masses (1 cm in diameter) located a little behind the preoperative point, namely the right internal obturator muscle.

proliferation of fibroblasts without the nuclear and cytoplasmic features of malignancy. They are locally aggressive and invade surrounding structures, but have no metastatic potential [2]. These tumors occur in $2.4-4.3$ individuals out of every million; $70-80 \%$ of patients are women [3]. The common age of patients with desmoid tumors reported in the literature is 20 - 30 years; the tumors occur especially during pregnancy and within 1 year after delivery [4].

Desmoid tumors are classified into abdominal or other types; the former can be divided into abdominal wall and intraabdominal desmoids. Tumors originating from the mesentery and pelvis are in the intra-abdominal group; thus, the present case belongs to this group. Although the etiology of desmoid tumors remains unknown, traumas including surgical incisions [3], APC gene mutations [5], and sex hormones [6] have been suggested as causal factors; this may explain the fact that $33 \%$ of desmoid tumors have been reported to have estrogen receptors [6], and there have been reported incidences of desmoid tumors during hormonal therapy for prostate cancer [7].

Surgery has historically been the primary treatment for patients with resectable desmoid tumors. Complete surgical resection of a tumor with negative microscopic margins is the gold surgical standard of care. Radiation therapy is used to treat patients with radiosensitive desmoid tumors not amenable to resection, local recurrence, or incompletely excised lesions. Chemotherapy and endocrine therapy have also been used to treat desmoid tumors in patients in whom resection is technically impossible because of a widespread tumor infiltration, and in patients who prefer conservative therapy to surgical therapy. Surrounding organs have been resected in many surgical cases; this has resulted in the occurrence of various symptoms. Dalen et al recommended non-surgical therapy for patients with no symptoms [8], and Sylvie et al for patients whose desmoid tumor size was $\square 7 \mathrm{~cm}$ [9]. Many studies have reported that medical therapies, mainly hormonal therapy and chemotherapy, were effective in the treatment of primary and recurrent desmoid tumors [10]. Moreover, many cases involving spontaneous regression of primary desmoid tumors have been reported $[9,11,12]$.

In the present case, there were recurrences after surgery, but no increase in tumor size occurred during pregnancy and after delivery; complete remission of the recurrent tumors occurred after only one simple observation. The relative decrease in estrogen density in the body after delivery, or other sex hormones, might have had an influence. To our knowledge, only one case has been reported regarding spontaneous regression 

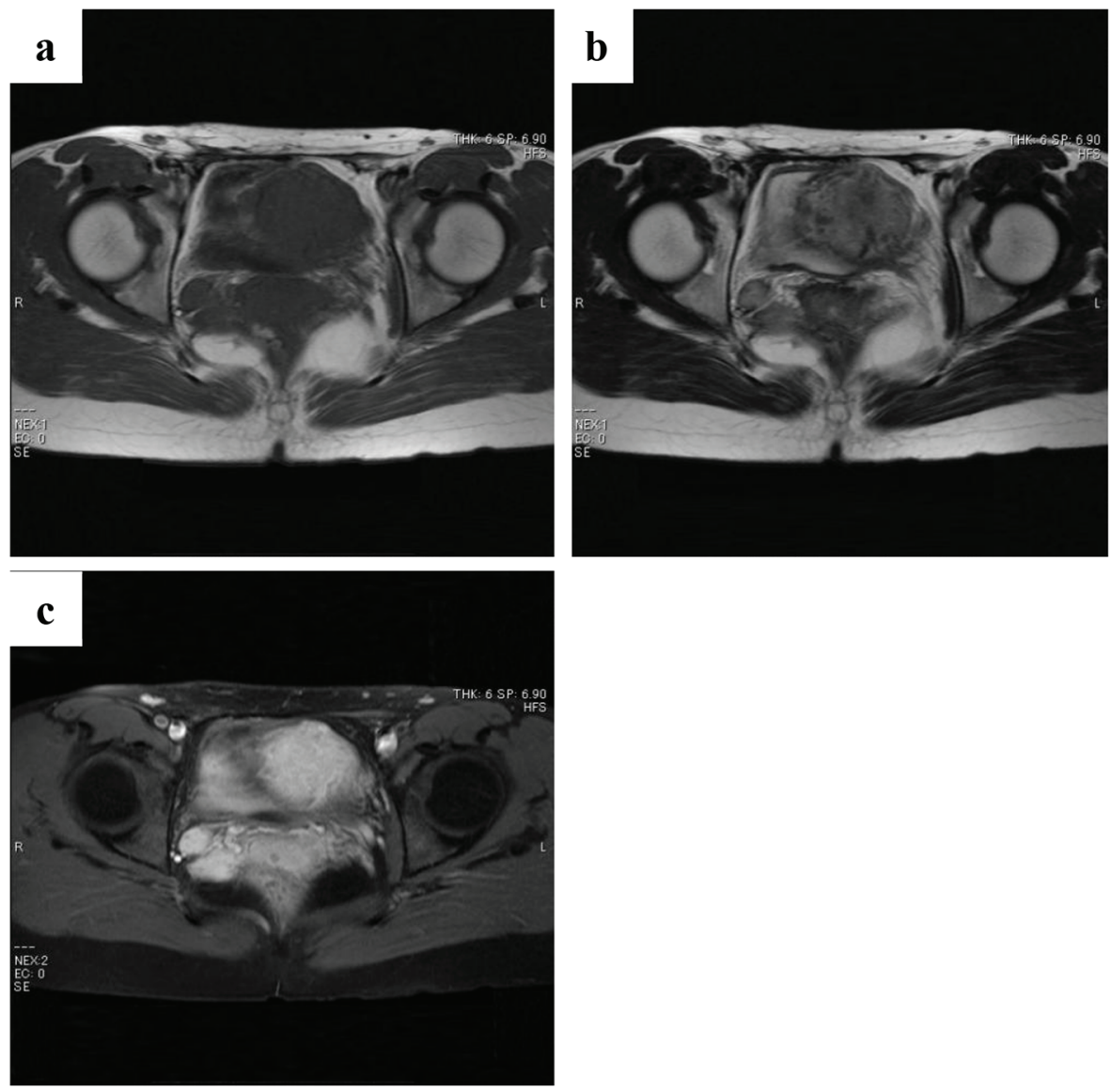

Figure 4. Magnetic resonance images of the two masses obtained at 3 years and 2 months after surgery (a: T1-weighted images; b: T2-weighted images; c: gadolinium contrast). These masses reached a maximum size of $1.8 \mathrm{~cm}$ in diameter.

of recurrent desmoid tumor following only simple observation [8]. The patient, who was diagnosed at 19 years of age, underwent resection involving a wide surgical margin because of the presence of a $7 \times 5 \mathrm{~cm}$ desmoid tumor in the abdominal wall; there was recurrence at 8 months after surgery. No further treatment was given, and the patient achieved complete remission of the tumor after postoperative follow-up at 5 and
21 years, as confirmed by magnetic resonance imaging. Our observations in the current case revealed a similar outcome. Our case is rare for two reasons. The first is that no increase in the size of the tumors occurred during pregnancy and after delivery, and the second is that the patient achieved complete remission after recurrence after only simple observation.

Desmoid tumors frequently exhibit self-limiting char-

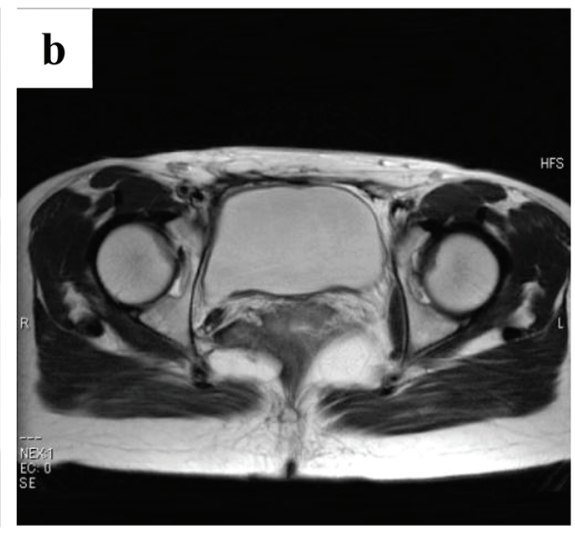

Figure 5. Magnetic resonance images of the two masses obtained at 5 years and 4 months after surgery (a: T1-weighted images; b: T2-weighted images). The masses have not increased in size. 

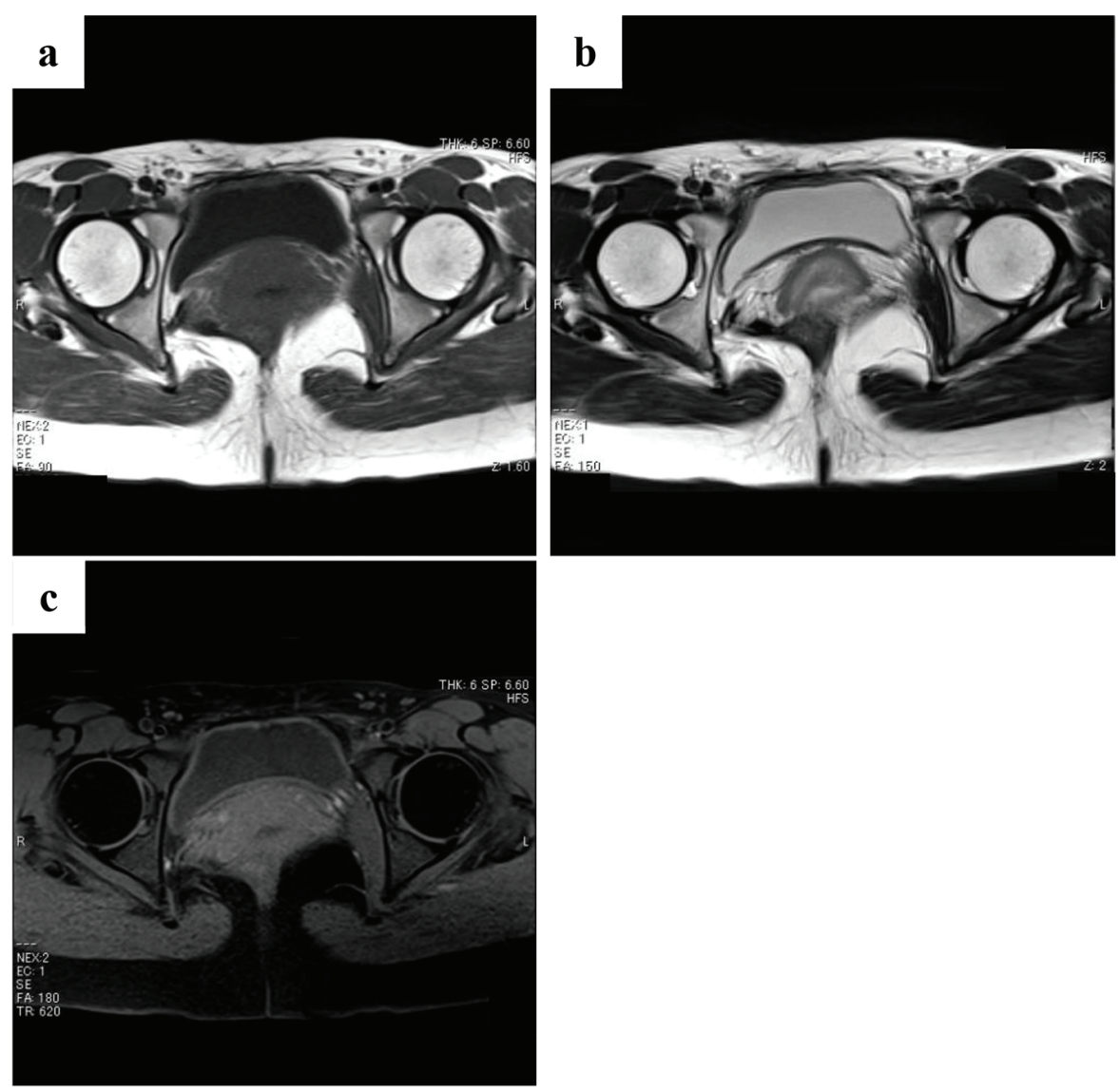

Figure 6. Magnetic resonance images obtained at 8 years and 4 months after surgery (a: T1-weighted images; b: T2-weighted images; c: gadolinium contrast). The enhanced masses have disappeared.

acteristics and growth arrest or spontaneous regression, especially primary cases $[8,13]$. These natural courses are not well known [12]. Simple observation involves function- and structure preserving therapy, so we will need to confirm its effectiveness in further studies regardless of the primary tumor or recurrence.

\section{Conflicts of Interest}

The authors declare that they have no conflicts of interest.

\section{References}

1. Enzinger, FM, Weiss SW. Fibromatoses: soft tissue tumors. St. Louis: CV Mosby Co; 1983.

2. Stout AP, Latters, R. Tumors of the soft tissues. Atlas of tumor pathology (second edition). Washington DC: Armed Forces Institute of Pathology; 1967.

3. Reitamo JJ, Hayry P, Nykyri E, Saxen E. The desmoid tumor. I. Incidence, sex-, age- and anatomical distribution in the Finnish population. Am J Clin Pathol. 1982;77(6):665-673.

4. Enzinger, FM and Weiss, SW. Fibromatoses. Soft tissue tumors (second edition). St. Louis: CV Mosby Co; 1988.

5. Turina M, Pavlik CM, Heinimann K, Behrensmeier F, Simmen HP. Recurrent desmoids determine outcome in patients with Gardner syndrome: a cohort study of three generations of an APC mutation-positive family across 30 years. Int J Colorectal Dis. 2013;28(6):865-872.

6. Lim CL, Walker MJ, Mehta RR, Das Gupta TK. Estrogen and antiestrogen binding sites in desmoid tumors. Eur J Cancer Clin Oncol. 1986;22(5):583-587.

7. Tokumo M, Mizoo T, Iga N, et al. A case of mesenteric desmoid tumor of the small intestine occurring during hormone therapy for prostate cancer. J Jpn Surg Assoc. 2010;71(12):3223-3226.

8. Dalen BP, Bergh PM, Gunterberg BU. Desmoid tumors: a clinical review of 30 patients with more than 20 years' follow-up. Acta Orthop Scand. 2003;74(4):455-459.

9. Bonvalot S, Ternes N, Fiore M, Bitsakou G, Colombo $\mathrm{C}$, Honore $\mathrm{C}$, Marrari A, et al. Spontaneous regression of primary abdominal wall desmoid tumors: more common than previously thought. Ann Surg Oncol. 2013;20(13):4096-4102.

10. Fiore M, Rimareix F, Mariani L, Domont J, Collini P, Le Pechoux C, Casali PG, et al. Desmoid-type fibromatosis: a front-line conservative approach to select patients for surgical treatment. Ann Surg Oncol. 2009;16(9):2587- 
2593.

11. Dalen BP, Geijer M, Kvist H, Bergh PM, Gunterberg BU. Clinical and imaging observations of desmoid tumors left without treatment. Acta Orthop. 2006;77(6):932-937.

12. Nakayama T, Tsuboyama T, Toguchida J, Hosaka T, Na- kamura T. Natural course of desmoid-type fibromatosis. J Orthop Sci. 2008;13(1):51-55.

13. Lewis JJ, Boland PJ, Leung DH, Woodruff JM, Brennan MF. The enigma of desmoid tumors. Ann Surg. 1999;229(6):866-872; discussion 872-863. 